\title{
The mixed boundary value problem for the inhomogeneous Cimmino system
}

\author{
Liping Wang ${ }^{1}$, Zuoliang $X \mathrm{u}^{2}$ and Yuying Qiao ${ }^{1 *}$
}

\section{"Correspondence:}

qiaoyuying@mail.hebtu.edu.cn

${ }^{1}$ College of Mathematics and Information Science, Hebei Normal

University, Shijiazhuang, Hebei province, 050024, P.R. China

Full list of author information is available at the end of the article

\begin{abstract}
In this article, we first propose a kind of mixed boundary value problem for the inhomogeneous Cimmino system, which consists of first order linear partial differential equations in $\mathbb{R}^{4}$. Then, by using the one-to-one correspondence between the theory of quaternion valued hyperholomorphic functions and that of Cimmino system's solutions, we transform the problem as stated above into a problem related to the $\psi$-hyperholomorphic functions in quaternionic analysis. Moreover, we show the boundedness, Hölder continuity, and generalized derivatives of a kind of singular integral operator ${ }^{\psi} T_{\mathbb{C}^{2}}[g]$ related to $\psi$-hyperholomorphic functions in quaternionic analysis. Lastly, the solution of the mixed boundary value problem for the inhomogeneous Cimmino system is explicitly described.
\end{abstract}

Keywords: Cimmino system; quaternionic analysis; $\psi$-hyperholomorphic functions; Cimmino singular integral operator; mixed boundary value problem

\section{Introduction}

The skew field of quaternions $\mathbb{H}$ gives an example of a noncommutative Clifford algebra with minimal dimension. It serves as a very convenient model of general Clifford constructions. Today, quaternionic analysis is regarded as a broadly accepted branch of classical analysis offering a successful generalization of complex analysis. It studies functions defined on domains in $\mathbb{R}^{3}$ or $\mathbb{R}^{4}$ with values in the skew field of real quaternions $\mathbb{H}$. This theory is centered around the concept of $\psi$-hyperholomorphic functions related to a socalled structural set $\psi$ of $\mathbb{H}^{3}$ or $\mathbb{H}^{4}$, respectively.

Quaternionic analysis initiated new solution methods for boundary value problems in several research areas of mathematical physics, in particular in planar fluids, quantum field theory, electromagnetic wave equations etc. Many scholars and experts have studied some boundary and initial value problems in higher dimensions by using them, such as Gürlebeck, Sprössig, Adler, Alesker, Yang, and so on [1-5].

The Cimmino system (1.1) offers a natural and elegant generalization to the fourdimensional case of that of Cauchy-Riemann. Cimmino, Dragomir and Lanconelli have done a lot of research on it [6, 7]. Recently, Abreu Blaya et al. [8] studied the Dirichlet boundary value problem for the inhomogeneous Cimmino system (1.2). We have

$$
\left\{\begin{array}{l}
\frac{\partial f_{0}}{\partial x_{0}}-\frac{\partial f_{1}}{\partial x_{1}}+\frac{\partial f_{2}}{\partial x_{2}}-\frac{\partial f_{3}}{\partial x_{3}}=0 \\
\frac{\partial f_{0}}{\partial x_{1}}+\frac{\partial f_{1}}{\partial x_{0}}-\frac{\partial f_{2}}{\partial x_{3}}-\frac{\partial f_{3}}{\partial x_{2}}=0 \\
\frac{\partial f_{0}}{\partial x_{2}}-\frac{\partial f_{1}}{\partial x_{3}}-\frac{\partial f_{2}}{\partial x_{0}}+\frac{\partial f_{3}}{\partial x_{1}}=0 \\
\frac{\partial f_{0}}{\partial x_{3}}+\frac{\partial f_{1}}{\partial x_{2}}+\frac{\partial f_{2}}{\partial x_{1}}+\frac{\partial f_{3}}{\partial x_{0}}=0
\end{array}\right.
$$


where $f_{m}(m=0,1,2,3)$ are continuously differentiable $\mathbb{R}$-valued functions in $\Omega \subset \mathbb{R}^{4}$. The corresponding inhomogeneous Cimmino system is as follows:

$$
\left\{\begin{array}{l}
\frac{\partial f_{0}}{\partial x_{0}}-\frac{\partial f_{1}}{\partial x_{1}}+\frac{\partial f_{2}}{\partial x_{2}}-\frac{\partial f_{3}}{\partial x_{3}}=g_{0}, \\
\frac{\partial f_{0}}{\partial x_{1}}+\frac{\partial f_{1}}{\partial x_{0}}-\frac{\partial f_{2}}{\partial x_{3}}-\frac{\partial f_{3}}{\partial x_{2}}=g_{1}, \\
\frac{\partial f_{0}}{\partial x_{2}}-\frac{\partial f_{1}}{\partial x_{3}}-\frac{\partial f_{2}}{\partial x_{0}}+\frac{\partial f_{3}}{\partial x_{1}}=g_{2}, \\
\frac{\partial f_{0}}{\partial x_{3}}+\frac{\partial f_{1}}{\partial x_{2}}+\frac{\partial f_{2}}{\partial x_{1}}+\frac{\partial f_{3}}{\partial x_{0}}=g_{3},
\end{array}\right.
$$

where $f_{m}$ are as stated above, $g_{m} \in L_{p}(\Omega, \mathbb{R})(m=0,1,2,3)$.

In this article, we will study a kind of mixed boundary value problem for the inhomogeneous Cimmino system (1.2) by using the quaternionic analysis approach. This article is organized as follows. In Section 2, we recall some basic knowledge of quaternionic analysis. In Section 3, we construct a singular integral operator and study some of its properties. In Section 4, we first propose a kind of mixed boundary value problem for the inhomogeneous Cimmino system (1.2); then we obtain an integral representation of the solution of the mixed boundary value problem by using the one-to-one correspondence between the theory of quaternion valued hyperholomorphic functions and that of a Cimmino system's solutions.

\section{Preliminaries}

Quaternionic analysis studies functions defined on $\mathbb{R}^{4}$ with their values in quaternion algebra space $\mathbb{H}$, which is a four-dimensional vector space with basis $e, i, j, k$. The basis element $e$ is a unit element, henceforth we shall abbreviate $e$ to 1 . Also, $i, j, k$ satisfy the following multiplication rule:

$$
i^{2}=j^{2}=k^{2}=-1, \quad i j=-j i=k, \quad j k=-k j=i, \quad k i=-i k=j .
$$

An arbitrary element of the quaternion algebra space $\mathbb{H}$ can be written as $x=x_{0}+i x_{1}+$ $j x_{2}+k x_{3}, x_{m} \in \mathbb{R}(m=0,1,2,3)$, and $\bar{x}=x_{0}-i x_{1}-j x_{2}-k x_{3}$. The norm for an element $x \in \mathbb{H}$ is taken to be $|x|=\sqrt{x_{0}^{2}+x_{1}^{2}+x_{2}^{2}+x_{3}^{2}}$ and satisfies $|\bar{x}|=|x|,|x+y| \leq|x|+|y|,|x y|=$ $|x||y|$. Obviously, $\overline{x y}=\bar{y} \bar{x}$ and $x \bar{x}=\bar{x} x=|x|^{2}$. In addition, suppose the imaginary unit of $\mathbb{C}$ is identified with the basis element $i$ in quaternion algebra space $\mathbb{H}$, then for arbitrary $z \in \mathbb{C}$, we have $z=x_{0}+i x_{1}$ and its complex conjugate $\bar{z}=x_{0}-i x_{1}$. In this way it is easily seen that $z j=j \bar{z}$.

By means of the mapping $x_{0}+i x_{1}+j x_{2}+k x_{3} \rightarrow\left(x_{0}+i x_{1}\right)+\left(x_{2}+i x_{3}\right) j\left(\rightarrow\left(x_{0}, x_{1}, x_{2}, x_{3}\right)\right)$, one can see $\mathbb{H}$ as $\mathbb{C}^{2}\left(\right.$ or $\left.\mathbb{R}^{4}\right)$. From now on, an arbitrary element $\xi \in \mathbb{H}$ can be written as $\xi=z_{1}+z_{2} j, z_{1}, z_{2} \in \mathbb{C}$. From the multiplication rule as stated above, for arbitrary $\xi=z_{1}+z_{2} j$, $\eta=\varsigma_{1}+\varsigma_{2} j \in \mathbb{H}, z_{1}, z_{2}, \varsigma_{1}, \varsigma_{2} \in \mathbb{C}$. We have $\xi \eta=\left(z_{1} \varsigma_{1}-z_{2} \bar{\zeta}_{2}\right)+\left(z_{1} \varsigma_{2}+z_{2} \bar{\varsigma}_{1}\right) j, \bar{\xi}=\overline{z_{1}+z_{2} j}=$ $\bar{z}_{1}+\overline{z_{2} j}=\bar{z}_{1}-z_{2} j$, and $\xi \bar{\xi}=\bar{\xi} \xi=\left|z_{1}\right|^{2}+\left|z_{2}\right|^{2}=|\xi|^{2}$.

Let $\Omega \subset \mathbb{R}^{4}$ be a nonempty open bounded connected set and the boundary $\Gamma=\partial \Omega$ be a differentiable, oriented, and compact Liapunov surface. The functions $f$ which are defined in $\Omega$ with values in $\mathbb{H}$ can be expressed as $f(x)=f_{0}+f_{1} i+f_{2} j+f_{3} k$, where $f_{m}(m=0,1,2,3)$ are continuously differentiable $\mathbb{R}$-valued functions in $\Omega \subset \mathbb{R}^{4}$. On $C^{(1)}(\Omega, \mathbb{H})$, we define the differential operators ${ }^{\psi} D$ and ${ }^{\bar{\psi}} D$ as follows:

$$
{ }^{\psi} D=2\left(\frac{\partial}{\partial \bar{z}_{1}}-j \frac{\partial}{\partial \bar{z}_{2}}\right), \quad{ }^{\bar{\psi}} D=2\left(\frac{\partial}{\partial z_{1}}+j \frac{\partial}{\partial \bar{z}_{2}}\right),
$$


where

$$
\begin{aligned}
\frac{\partial}{\partial \bar{z}_{1}}=\frac{1}{2}\left(\frac{\partial}{\partial x_{0}}+i \frac{\partial}{\partial x_{1}}\right), & \frac{\partial}{\partial \bar{z}_{2}}=\frac{1}{2}\left(\frac{\partial}{\partial x_{2}}+i \frac{\partial}{\partial x_{3}}\right), \\
\frac{\partial}{\partial z_{1}}=\frac{1}{2}\left(\frac{\partial}{\partial x_{0}}-i \frac{\partial}{\partial x_{1}}\right), & \frac{\partial}{\partial z_{2}}=\frac{1}{2}\left(\frac{\partial}{\partial x_{2}}-i \frac{\partial}{\partial x_{3}}\right) .
\end{aligned}
$$

Obviously, the differential operators ${ }^{\psi} D$ and ${ }^{\bar{\psi}} D$ can be written as

$$
{ }^{\psi} D=\frac{\partial}{\partial x_{0}}+i \frac{\partial}{\partial x_{1}}-j \frac{\partial}{\partial x_{2}}+k \frac{\partial}{\partial x_{3}}, \quad{ }^{\bar{\psi}} D=\frac{\partial}{\partial x_{0}}-i \frac{\partial}{\partial x_{1}}+j \frac{\partial}{\partial x_{2}}-k \frac{\partial}{\partial x_{3}},
$$

which are associated to the structural set $\psi=\{1, i,-j, k\}$ and $\bar{\psi}=\{1,-i, j,-k\}$, respectively. Let $\Delta_{\mathbb{R}^{4}}=\sum_{m=0}^{3} \partial_{x_{m}}^{2}$, then the following equalities hold on $C^{(2)}(\Omega, \mathbb{H})$ :

$$
{ }^{\psi} D^{\bar{\psi}} D={ }^{\bar{\psi}} D^{\psi} D=\Delta_{\mathbb{R}^{4}} \cong \Delta_{\mathbb{C}^{2}} \cong \Delta_{\mathbb{H}} \cdot
$$

Taking into account that the multiplication in $\mathbb{H}$ is noncommutative, the functions $f$ are called left $\psi$-hyperholomorphic in $\Omega$ if ${ }^{\psi} D[f](\xi)=0(\xi \in \Omega)$. The functions $g$ are called right $\psi$-hyperholomorphic in $\Omega$ if $[g]^{\psi} D(\xi)=0(\xi \in \Omega)$.

Denote by $\Theta_{4}$ the fundamental solution of the Laplace operator

$$
\Theta_{4}(\xi)=-\frac{1}{4 \pi^{2}} \frac{1}{|\xi|^{2}}
$$

and by $\mathcal{K}_{\psi}$ the fundamental solution of the operator ${ }^{\psi} D$ :

$$
\mathcal{K}_{\psi}(\xi)={ }^{\bar{\psi}} D\left[\Theta_{4}\right]=\left[\Theta_{4}\right]^{\bar{\psi}} D=\frac{1}{2 \pi^{2}} \frac{\xi_{\bar{\psi}}}{|\xi|^{4}}=\frac{1}{2 \pi^{2}} \frac{\bar{z}_{1}+\bar{z}_{2} j}{\left(\left|z_{1}\right|^{2}+\left|z_{2}\right|^{2}\right)^{2}} .
$$

Then the corresponding Cauchy type integral operator is

$$
{ }^{\psi} K_{\Gamma}[f](\xi)=\int_{\Gamma} \mathcal{K}_{\psi}(\eta-\xi) n_{\psi}(\eta) f(\eta) d \Gamma_{\eta}=\int_{\Gamma} \mathcal{K}_{\psi}(\eta-\xi) d \sigma_{\eta} f(\eta),
$$

and the Teodorescu type integral operator is

$$
{ }^{\psi} T_{\Omega}[f](\xi)=-\int_{\Omega} \mathcal{K}_{\psi}(\eta-\xi) f(\eta) d \Omega_{\eta}
$$

In this article, $g(x) \in L_{p}\left(\mathbb{C}^{2}, \mathbb{H}\right)$ means that $g(x) \in L_{p}(E, \mathbb{H}), g_{\sigma}(x)=|x|^{-\sigma} g\left(\frac{\bar{x}}{|x|^{2}}\right) \in L_{p}(E, \mathbb{H})$, in which $E=\{\xi|| \xi \mid \leq 1\}, \sigma$ is a real number, $\|g\|_{L_{p}}=\|g\|_{L_{p}(E)}+\left\|g_{\sigma}\right\|_{L_{p}(E)}, p \geq 1$. The following fundamental statements are widely known to hold and can be found in $[1,9,10]$, respectively.

Definition 2.1 Suppose that the functions $f, g, \varphi$ are defined in $\Omega$ with values in $\mathbb{H}$ and $f, g \in L_{1}(\Omega, \mathbb{H})$. If for arbitrary $\varphi \in C_{0}^{\infty}(\Omega, \mathbb{H}), f, g$ satisfy

$$
\int_{\Omega}[\varphi]^{\psi} D(\xi) g(\xi) d \Omega_{\xi}+\int_{\Omega} \varphi(\xi) f(\xi) d \Omega_{\xi}=0
$$

then $f$ is called a generalized derivative of the function $g$, denoted by $f={ }^{\psi} D[g]$. 
Lemma 2.1 ([9]) If $\sigma_{1}, \sigma_{2}>0,0 \leq \gamma \leq 1$, then we have

$$
\left|\sigma_{1}^{\gamma}-\sigma_{2}^{\gamma}\right| \leq\left|\sigma_{1}-\sigma_{2}\right|^{\gamma}
$$

Lemma 2.2 (Integral form of the quaternionic Stokes formula [1]) Let $\Omega, \Gamma=\partial \Omega$ be as stated above and $f, g \in C^{(1)}(\Omega, \mathbb{H})$, then

$$
\int_{\Gamma} g(\xi) n_{\psi}(\xi) f(\xi) d \Gamma_{\xi}=\int_{\Omega}\left([g]^{\psi} D(\xi) \cdot f(\xi)+g(\xi) \cdot{ }^{\psi} D[f](\xi)\right) d \Omega_{\xi} .
$$

Lemma 2.3 (Borel-Pompeiu quaternionic formula [1]) Let $\Omega, \Gamma=\partial \Omega$ be as stated above and $f \in C^{(1)}(\Omega, \mathbb{H})$, then for arbitrary $\xi \in \Omega$, we have

$$
\int_{\Gamma} \mathcal{K}_{\psi}(\eta-\xi) d \sigma_{\eta} f(\eta)-\int_{\Omega} \mathcal{K}_{\psi}(\eta-\xi)^{\psi} D[f](\eta) d_{\Omega_{\eta}}=f(\xi)
$$

and

$$
\int_{\Gamma} f(\eta) d \sigma_{\eta} \mathcal{K}_{\psi}(\eta-\xi)-\int_{\Omega}[f]^{\psi} D(\eta) \mathcal{K}_{\psi}(\eta-\xi) d_{\Omega_{\eta}}=f(\xi) .
$$

Lemma 2.4 (Hadamard lemma [10]) Suppose $\Omega$ be as stated above. If $\alpha^{\prime}, \beta^{\prime}$ satisfy $0<$ $\alpha^{\prime}, \beta^{\prime}<4, \alpha^{\prime}+\beta^{\prime}>4$, then for all $x_{1}, x_{2} \in \mathbb{R}^{4}$ and $x_{1} \neq x_{2}$, we have

$$
\int_{\Omega}\left|t-x_{1}\right|^{-\alpha^{\prime}}\left|t-x_{2}\right|^{-\beta^{\prime}} d t \leq M_{0}\left(\alpha^{\prime}, \beta^{\prime}\right)\left|x_{1}-x_{2}\right|^{4-\alpha^{\prime}-\beta^{\prime}} .
$$

\section{Some useful properties of the Cimmino singular integral operator}

By means of the idea as stated above, we suppose

$$
\begin{aligned}
& \xi=z_{1}+z_{2} j \in \mathbb{H}, \quad z_{1}=x_{0}+i x_{1}, z_{2}=x_{2}+i x_{3} \in \mathbb{C}, \\
& \eta=\varsigma_{1}+\varsigma_{2} j \in \mathbb{H}, \quad \varsigma_{1}=y_{0}+i y_{1}, \varsigma_{2}=y_{2}+i y_{3} \in \mathbb{C}, \\
& f(\xi)=f\left(z_{1}, z_{2}\right)=u_{1}\left(z_{1}, z_{2}\right)+u_{2}\left(z_{1}, z_{2}\right) j, \quad u_{1}=f_{0}+i f_{1}, u_{2}=f_{2}+i f_{3} \in \mathbb{C}, \\
& g(\xi)=g\left(z_{1}, z_{2}\right)=v_{1}\left(z_{1}, z_{2}\right)+v_{2}\left(z_{1}, z_{2}\right) j, \quad v_{1}=g_{0}+i g_{1}, v_{2}=g_{2}+i g_{3} \in \mathbb{C} .
\end{aligned}
$$

Then system (1.1) can be written as

$$
(1.1) \Longleftrightarrow\left\{\begin{array}{l}
\partial_{\bar{z}_{1}} u_{1}+\partial_{z_{2}} \bar{u}_{2}=0, \\
\partial_{\bar{z}_{2}} u_{1}-\partial_{z_{1}} \bar{u}_{2}=0
\end{array} \quad \Longleftrightarrow{ }^{\psi} D[f]=0 .\right.
$$

Moreover, if the pair $\left(u_{1}, u_{2}\right)$ of continuously differentiable (up to the second order) complex-valued functions give a solution of system (1.1) then

$$
\Delta_{\mathbb{R}^{4}} u_{1} \cong \Delta_{\mathbb{C}^{2}} u_{1}=4\left(\partial_{z_{1} \bar{z}_{1}}^{2}+\partial_{z_{2} \bar{z}_{2}}^{2}\right) u_{1}=0
$$

A similar observation is valid for $\bar{u}_{2}$, so $u_{1}, \bar{u}_{2}$ are complex-valued harmonic functions, i.e. the set of solutions of system (1.1) contains all holomorphic functions of two complex variables. 
Similarly, system (1.2) can be written as

$$
(1.2) \quad \Longleftrightarrow\left\{\begin{array}{l}
2 \partial_{\bar{z}_{1}} u_{1}+2 \partial_{z_{2}} \bar{u}_{2}=v_{1}, \\
2 \partial_{\bar{z}_{2}} u_{1}-2 \partial_{z_{1}} \bar{u}_{2}=v_{2}
\end{array} \quad \Longleftrightarrow \quad \psi D[f]=g\right.
$$

The generalized Teodorescu type integral operator ${ }^{\psi} T_{\mathbb{C}^{2}}[g]$ can be written as

$$
\begin{aligned}
{ }^{\psi} T_{\mathbb{C}^{2}}[g]\left(z_{1}, z_{2}\right) & ={ }^{\psi} T_{\mathbb{C}^{2}}[g](\xi) \\
& =-\int_{\mathbb{C}^{2}} \mathcal{K}_{\psi}(\eta-\xi) g(\eta) d_{\mathbb{C}^{2} \eta} \\
& =\frac{1}{2 \pi^{2}} \int_{\mathbb{C}^{2}} \frac{\left(\bar{z}_{1}-\bar{\zeta}_{1}\right)+\left(\bar{z}_{2}-\bar{\zeta}_{2}\right) j}{\left(\left|z_{1}-\varsigma_{1}\right|^{2}+\left|z_{2}-\varsigma_{2}\right|^{2}\right)^{2}} g\left(\varsigma_{1}, \varsigma_{2}\right) d_{\mathbb{C}^{2}{ }_{\varsigma_{1}, \varsigma_{2}}} \\
& ={ }^{\psi} T_{\mathbb{C}^{2}}^{(1)}[g]\left(z_{1}, z_{2}\right)+{ }^{\psi} T_{\mathbb{C}^{2}}^{(2)}[g]\left(z_{1}, z_{2}\right) j,
\end{aligned}
$$

where the Cimmino singular integral operators ${ }^{\psi} T_{\mathbb{C}^{2}}^{(1)}[g],{ }^{\psi} T_{\mathbb{C}^{2}}^{(2)}[g]$ are as follows:

$$
\begin{aligned}
& { }^{\psi} T_{\mathbb{C}^{2}}^{(1)}[g]\left(z_{1}, z_{2}\right)=\frac{1}{2 \pi^{2}} \int_{\mathbb{C}^{2}} \frac{\left(\bar{z}_{1}-\bar{\zeta}_{1}\right)}{\left(\left|z_{1}-\varsigma_{1}\right|^{2}+\left|z_{2}-\varsigma_{2}\right|^{2}\right)^{2}} g\left(\varsigma_{1}, \varsigma_{2}\right) d_{\mathbb{C}^{2}{ }_{\varsigma_{1}, \varsigma_{2}}}, \\
& { }^{\psi} T_{\mathbb{C}^{2}}^{(2)}[g]\left(z_{1}, z_{2}\right)=\frac{1}{2 \pi^{2}} \int_{\mathbb{C}^{2}} \frac{\left(\bar{z}_{2}-\bar{\zeta}_{2}\right)}{\left(\left|z_{1}-\varsigma_{1}\right|^{2}+\left|z_{2}-\varsigma_{2}\right|^{2}\right)^{2}} g\left(\varsigma_{1}, \varsigma_{2}\right) d_{\mathbb{C}^{2}{ }_{\varsigma_{1}, \varsigma_{2}}}
\end{aligned}
$$

Theorem 3.1 Let $E$ be as stated above. If $g \in L_{p}\left(\mathbb{C}^{2}, \mathbb{H}\right), 4<p<+\infty$, then we have

(1) $\left|{ }^{\psi} T_{\mathbb{C}^{2}}[g](\xi)\right| \leq M_{1}(p)\|g\|_{L_{p}}, \xi \in \mathbb{C}^{2} \cong \mathbb{R}^{4}$,

(2) ${ }^{\psi} T_{\mathbb{C}^{2}}[g] \in C_{\beta}\left(\mathbb{C}^{2}, \mathbb{H}\right) \cong C_{\beta}\left(\mathbb{R}^{4}, \mathbb{H}\right)(0<\beta=1-4 / p<1)$,

(3) ${ }^{\psi} D\left({ }^{\psi} T_{\mathbb{C}^{2}}[g]\right)(\xi)=g(\xi), \xi \in \mathbb{C}^{2} \cong \mathbb{R}^{4}$.

Proof (1) First, we have

$$
\begin{aligned}
\left|{ }^{\psi} T_{\mathbb{C}^{2}}[g](\xi)\right| \leq & \frac{1}{2 \pi^{2}}\left|\int_{E} \frac{\left(\bar{z}_{1}-\bar{\zeta}_{1}\right)+\left(\bar{z}_{2}-\bar{\zeta}_{2}\right) j}{\left(\left|z_{1}-\varsigma_{1}\right|^{2}+\left|z_{2}-\varsigma_{2}\right|^{2}\right)^{2}} g(\eta) d E_{\eta}\right| \\
& +\frac{1}{2 \pi^{2}}\left|\int_{\mathbb{C}^{2}-E} \frac{\left(\bar{z}_{1}-\bar{\zeta}_{1}\right)+\left(\bar{z}_{2}-\bar{\zeta}_{2}\right) j}{\left(\left|z_{1}-\varsigma_{1}\right|^{2}+\left|z_{2}-\varsigma_{2}\right|^{2}\right)^{2}} g(\eta) d_{\left(\mathbb{C}^{2}-E\right)_{\eta}}\right| \\
= & O_{1}+O_{2} .
\end{aligned}
$$

By the Hölder inequality, we have

$$
\begin{aligned}
O_{1} & =\frac{1}{2 \pi^{2}}\left|\int_{E} \frac{\left(\bar{z}_{1}-\bar{\zeta}_{1}\right)+\left(\bar{z}_{2}-\bar{\zeta}_{2}\right) j}{\left(\left|z_{1}-\varsigma_{1}\right|^{2}+\left|z_{2}-\varsigma_{2}\right|^{2}\right)^{2}} g(\eta) d E_{\eta}\right| \\
& \leq \frac{1}{2 \pi^{2}} \int_{E} \frac{\left|z_{1}-\varsigma_{1}\right|+\left|z_{2}-\varsigma_{2}\right|}{|\xi-\eta|^{4}}|g(\eta)| d E_{\eta} \\
& \leq \frac{1}{\pi^{2}} \int_{E} \frac{1}{|\xi-\eta|^{3}}|g(\eta)| d E_{\eta} \leq \frac{1}{\pi^{2}}\|g\|_{L_{p}}\left(\int_{E} \frac{1}{|\xi-\eta|^{3 q}} d E_{\eta}\right)^{\frac{1}{q}}
\end{aligned}
$$

where $1 / p+1 / q=1$. 
When $\xi \in \bar{E}$, because of $4<p<+\infty, 1 / p+1 / q=1$, we have $1<q<4 / 3$. Thus $\int_{E} \frac{1}{|\xi-\eta|^{3 q}} d E_{\eta}$ is bounded. Hence we have

$$
\left(\int_{E} \frac{1}{|\xi-\eta|^{3 q}} d E_{\eta}\right)^{\frac{1}{q}} \leq J_{1}
$$

When $\xi \in \mathbb{C}^{2}-\bar{E}$, by Lemma 2.1 and the generalized spherical coordinate, we have

$$
\left(\int_{E} \frac{1}{|\xi-\eta|^{3 q}} d E_{\eta}\right)^{\frac{1}{q}} \leq J_{2}\left(\int_{d_{0}}^{d_{0}+2} \rho^{3-3 q} d \rho\right)^{\frac{1}{q}} \leq J_{3},
$$

where $\rho=|\xi-\eta|, d_{0}=d(\xi, \bar{E})$.

Therefore, for $\forall \xi \in \mathbb{C}^{2} \cong \mathbb{R}^{4}$, we can obtain

$$
O_{1} \leq M_{1}^{\prime}(p)\|g\|_{L_{p}}, \quad \xi \in \mathbb{C}^{2} \cong \mathbb{R}^{4},
$$

where $M_{1}^{\prime}(p)=\max \left\{J_{1} / \pi^{2}, J_{3} / \pi^{2}\right\}$.

For $\eta \in \mathbb{C}^{2}-E$, we suppose that $\eta=\frac{\bar{\eta}^{\prime}}{\left|\eta^{\prime}\right|^{2}}$, then we have $\left|\eta^{\prime}\right| \leq 1$. Thus by $g \in L_{p}\left(\mathbb{C}^{2}, \mathbb{H}\right)$, similar to the proof as stated above, we have

$$
O_{2} \leq M_{1}^{\prime \prime}(p)\|g\|_{L_{p}} .
$$

Therefore, we obtain

$$
\left|{ }^{\psi} T_{\mathbb{C}^{2}}[g](\xi)\right| \leq M_{1}(p)\|g\|_{L_{p}}, \quad \xi \in \mathbb{C}^{2} \cong \mathbb{R}^{4},
$$

where $M_{1}(p)=M_{1}^{\prime}(p)+M_{1}^{\prime \prime}(p)$.

(2) For arbitrary $\xi^{\prime}, \xi^{\prime \prime} \in \mathbb{C}^{2} \cong \mathbb{R}^{4}, \xi^{\prime} \neq \xi^{\prime \prime}$, we have

$$
\begin{aligned}
{ }^{\psi} T_{\mathbb{C}^{2}}[g]\left(\xi^{\prime}\right)-{ }^{\psi} T_{\mathbb{C}^{2}}[g]\left(\xi^{\prime \prime}\right) \mid & =\frac{1}{2 \pi^{2}}\left|\int_{\mathbb{C}^{2}}\left[\frac{\left(\bar{z}_{1}^{\prime}-\bar{\zeta}_{1}\right)+\left(\bar{z}_{2}^{\prime}-\bar{\zeta}_{2}\right) j}{\left|\xi^{\prime}-\eta\right|^{4}}-\frac{\left(\bar{z}_{1}^{\prime \prime}-\bar{\zeta}_{1}\right)+\left(\bar{z}_{2}^{\prime \prime}-\bar{\zeta}_{2}\right) j}{\left|\xi^{\prime \prime}-\eta\right|^{4}}\right] g(\eta) d_{\mathbb{C}^{2} \eta}\right| \\
\leq & \frac{1}{2 \pi^{2}}\left|\int_{E}\left[\frac{\left(\bar{z}_{1}^{\prime}-\bar{\zeta}_{1}\right)+\left(\bar{z}_{2}^{\prime}-\bar{\zeta}_{2}\right) j}{\left|\xi^{\prime}-\eta\right|^{4}}-\frac{\left(\bar{z}_{1}^{\prime \prime}-\bar{\zeta}_{1}\right)+\left(\bar{z}_{2}^{\prime \prime}-\bar{\zeta}_{2}\right) j}{\left|\xi^{\prime \prime}-\eta\right|^{4}}\right] g(\eta) d E_{\eta}\right| \\
& +\frac{1}{2 \pi^{2}}\left|\int_{\mathbb{C}^{2}-E}\left[\frac{\left(\bar{z}_{1}^{\prime}-\bar{\zeta}_{1}\right)+\left(\bar{z}_{2}^{\prime}-\bar{\zeta}_{2}\right) j}{\left|\xi^{\prime}-\eta\right|^{4}}-\frac{\left(\bar{z}_{1}^{\prime \prime}-\bar{\zeta}_{1}\right)+\left(\bar{z}_{2}^{\prime \prime}-\bar{\zeta}_{2}\right) j}{\left|\xi^{\prime \prime}-\eta\right|^{4}}\right] g(\eta) d_{\left(\mathbb{C}^{2}-E\right)_{\eta}}\right| \\
= & O_{3}+O_{4}
\end{aligned}
$$

and

$$
\begin{aligned}
O_{3} \leq & \frac{1}{2 \pi^{2}}\left|\int_{E}\left[\frac{\left(\bar{z}_{1}^{\prime}-\bar{\zeta}_{1}\right)}{\left|\xi^{\prime}-\eta\right|^{4}}-\frac{\left(\bar{z}_{1}^{\prime \prime}-\bar{\zeta}_{1}\right)}{\left|\xi^{\prime \prime}-\eta\right|^{4}}\right] g(\eta) d E_{\eta}\right| \\
& +\frac{1}{2 \pi^{2}}\left|\iint_{E}\left[\frac{\left(\bar{z}_{2}^{\prime}-\bar{\zeta}_{2}\right) j}{\left|\xi^{\prime}-\eta\right|^{4}}-\frac{\left(\bar{z}_{2}^{\prime \prime}-\bar{\zeta}_{2}\right) j}{\left|\xi^{\prime \prime}-\eta\right|^{4}}\right] g(\eta) d E_{\eta}\right| \\
= & \left|{ }^{\psi} T_{E}^{(1)}[g]\left(z_{1}^{\prime}, z_{2}^{\prime}\right)-{ }^{\psi} T_{E}^{(1)}[g]\left(z_{1}^{\prime \prime}, z_{2}^{\prime \prime}\right)\right|+\left|{ }^{\psi} T_{E}^{(2)}[g]\left(z_{1}^{\prime}, z_{2}^{\prime}\right)-{ }^{\psi} T_{E}^{(2)}[g]\left(z_{1}^{\prime \prime}, z_{2}^{\prime \prime}\right)\right| \\
= & I_{1}+I_{2} .
\end{aligned}
$$


Since

$$
\begin{aligned}
\left|\frac{\bar{z}_{1}^{\prime}-\bar{\zeta}_{1}}{\left|\xi^{\prime}-\eta\right|^{4}}-\frac{\bar{z}_{1}^{\prime \prime}-\bar{\zeta}_{1}}{\left|\xi^{\prime \prime}-\eta\right|^{4}}\right| & \mid \frac{\left(\bar{z}_{1}^{\prime}-\bar{\zeta}_{1}\right)\left|\xi^{\prime \prime}-\eta\right|^{2}\left(\left|z_{1}^{\prime \prime}-\varsigma_{1}\right|^{2}+\left|z_{2}^{\prime \prime}-\varsigma_{2}\right|^{2}\right)}{\left|\xi^{\prime}-\eta\right|^{4}\left|\xi^{\prime \prime}-\eta\right|^{4}} \\
= & \quad-\frac{\left(\left|z_{1}^{\prime}-\varsigma_{1}\right|^{2}+\left|z_{2}^{\prime}-\varsigma_{2}\right|^{2}\right)\left|\xi^{\prime}-\eta\right|^{2}\left(\bar{z}_{1}^{\prime \prime}-\bar{\zeta}_{1}\right) \mid}{\left|\xi^{\prime}-\eta\right|^{4}\left|\xi^{\prime \prime}-\eta\right|^{4}} \mid \\
\leq & \frac{\left|\left(\bar{z}_{1}^{\prime}-\bar{\zeta}_{1}\right)\right| \xi^{\prime \prime}-\left.\eta\right|^{2}\left|z_{1}^{\prime \prime}-\varsigma_{1}\right|^{2}-\left|z_{1}^{\prime}-\varsigma_{1}\right|^{2}\left|\xi^{\prime}-\eta\right|^{2}\left(\bar{z}_{1}^{\prime \prime}-\bar{\zeta}_{1}\right) \mid}{\left|\xi^{\prime}-\eta\right|^{4}\left|\xi^{\prime \prime}-\eta\right|^{4}} \\
& +\frac{\left|\left(\bar{z}_{1}^{\prime}-\bar{\zeta}_{1}\right)\right| \xi^{\prime \prime}-\left.\eta\right|^{2}\left|z_{2}^{\prime \prime}-\varsigma_{2}\right|^{2}-\left|z_{2}^{\prime}-\varsigma_{2}\right|^{2}\left|\xi^{\prime}-\eta\right|^{2}\left(\bar{z}_{1}^{\prime \prime}-\bar{\zeta}_{1}\right) \mid}{\left|\xi^{\prime}-\eta\right|^{4}\left|\xi^{\prime \prime}-\eta\right|^{4}} \\
= & \mathcal{K}_{1}\left(\xi^{\prime}, \xi^{\prime \prime}, \eta\right)+\mathcal{K}_{2}\left(\xi^{\prime}, \xi^{\prime \prime}, \eta\right) .
\end{aligned}
$$

Thus

$$
I_{1} \leq \frac{1}{2 \pi^{2}} \int_{E} \mathcal{K}_{1}\left(\xi^{\prime}, \xi^{\prime \prime}, \eta\right)|g(\eta)| d E_{\eta}+\frac{1}{2 \pi^{2}} \int_{E} \mathcal{K}_{2}\left(\xi^{\prime}, \xi^{\prime \prime}, \eta\right)|g(\eta)| d E_{\eta}=I_{1_{1}}+I_{1_{2}} .
$$

Again, because of

$$
\begin{aligned}
\mathcal{K}_{1}\left(\xi^{\prime}, \xi^{\prime \prime}, \eta\right) & \\
& =\frac{\left|\left(\bar{z}_{1}^{\prime}-\bar{\zeta}_{1}\right)\right| \xi^{\prime \prime}-\left.\eta\right|^{2}\left|z_{1}^{\prime \prime}-\varsigma_{1}\right|^{2}-\left|z_{1}^{\prime}-\varsigma_{1}\right|^{2}\left|\xi^{\prime}-\eta\right|^{2}\left(\bar{z}_{1}^{\prime \prime}-\bar{\zeta}_{1}\right) \mid}{\left|\xi^{\prime}-\eta\right|^{4}\left|\xi^{\prime \prime}-\eta\right|^{4}} \\
& =\frac{\left|\left(\bar{z}_{1}^{\prime}-\bar{\zeta}_{1}\right)\right| \xi^{\prime \prime}-\left.\eta\right|^{2}\left(z_{1}^{\prime \prime}-\varsigma_{1}\right)\left(\bar{z}_{1}^{\prime \prime}-\bar{\zeta}_{1}\right)-\left(\bar{z}_{1}^{\prime}-\bar{\zeta}_{1}\right)\left(z_{1}^{\prime}-\varsigma_{1}\right)\left|\xi^{\prime}-\eta\right|^{2}\left(\bar{z}_{1}^{\prime \prime}-\bar{\zeta}_{1}\right) \mid}{\left|\xi^{\prime}-\eta\right|^{4}\left|\xi^{\prime \prime}-\eta\right|^{4}} \\
& =\frac{\left|\bar{z}_{1}^{\prime}-\bar{\zeta}_{1}\right||| \xi^{\prime \prime}-\left.\eta\right|^{2}\left(z_{1}^{\prime \prime}-\varsigma_{1}\right)-\left(z_{1}^{\prime}-\varsigma_{1}\right)\left|\xi^{\prime}-\eta\right|^{2}|| \bar{z}_{1}^{\prime \prime}-\bar{\zeta}_{1} \mid}{\left|\xi^{\prime}-\eta\right|^{4}\left|\xi^{\prime \prime}-\eta\right|^{4}} \\
& \leq \frac{\left|\bar{z}_{1}^{\prime}-\bar{\zeta}_{1}\right|\left[\left|\xi^{\prime \prime}-\eta\right|^{2}\left|z_{1}^{\prime \prime}-z_{1}^{\prime}\right|+|| \xi^{\prime \prime}-\left.\eta\right|^{2}-\left|\xi^{\prime}-\eta\right|^{2}|| z_{1}^{\prime}-\varsigma_{1} \mid\right]\left|\bar{z}_{1}^{\prime \prime}-\bar{\zeta}_{1}\right|}{\left|\xi^{\prime}-\eta\right|^{4}\left|\xi^{\prime \prime}-\eta\right|^{4}} \\
& \leq \frac{\left|\bar{z}_{1}^{\prime}-\bar{\zeta}_{1}\right|\left[\left|\xi^{\prime \prime}-\eta\right|^{2}\left|z_{1}^{\prime \prime}-z_{1}^{\prime}\right|+\left|\xi^{\prime \prime}-\xi^{\prime}\right|\left(\left|\xi^{\prime \prime}-\eta\right|+\left|\xi^{\prime}-\eta\right|\right)\left|z_{1}^{\prime}-\varsigma_{1}\right|\right]\left|\bar{z}_{1}^{\prime \prime}-\bar{\zeta}_{1}\right|}{\left|\xi^{\prime}-\eta\right|^{4}\left|\xi^{\prime \prime}-\eta\right|^{4}} \\
& \leq \frac{\left|\xi^{\prime}-\eta\right|\left[\left|\xi^{\prime \prime}-\eta\right|^{2}\left|\xi^{\prime \prime}-\xi^{\prime}\right|+\left|\xi^{\prime \prime}-\xi^{\prime}\right|\left(\left|\xi^{\prime \prime}-\eta\right|+\left|\xi^{\prime}-\eta\right|\right)\left|\xi^{\prime}-\eta\right|\right]\left|\xi^{\prime \prime}-\eta\right|}{\left|\xi^{\prime}-\eta\right|^{4}\left|\xi^{\prime \prime}-\eta\right|^{4}} \\
& =\left|\xi^{\prime}-\xi^{\prime \prime}\right| \sum_{l=1}^{3} \frac{1}{\left|\xi^{\prime}-\eta\right|^{4-l}\left|\xi^{\prime \prime}-\eta\right|^{l}} .
\end{aligned}
$$

Thus by (3.5), (3.6), and the Hölder inequality, we have

$$
\begin{aligned}
I_{1_{1}} & \leq \frac{1}{2 \pi^{2}} \int_{E} \sum_{l=1}^{3} \frac{1}{\left|\xi^{\prime}-\eta\right|^{4-l}\left|\xi^{\prime \prime}-\eta\right|^{l}}|g(\eta)| d E_{\eta}\left|\xi^{\prime}-\xi^{\prime \prime}\right| \\
& \leq \frac{1}{2 \pi^{2}}\|g\|_{L_{p}}\left|\xi^{\prime}-\xi^{\prime \prime}\right| \sum_{l=1}^{3}\left(\int_{E} \frac{1}{\left|\xi^{\prime}-\eta\right|^{(4-l) q}\left|\xi^{\prime \prime}-\eta\right|^{l q}} d E_{\eta}\right)^{\frac{1}{q}} \\
& =\frac{1}{2 \pi^{2}}\|g\|_{L_{p}}\left|\xi^{\prime}-\xi^{\prime \prime}\right| \sum_{l=1}^{3}\left(I_{1_{1}}^{(l)}\right)^{\frac{1}{q}} .
\end{aligned}
$$


Suppose $\alpha_{l}=(4-l) q, \beta_{l}=l q(l=1,2,3)$. By $1<q<4 / 3$, we know

$$
0<\alpha_{l}, \beta_{l}<4, \quad \alpha_{l}+\beta_{l}=4 q>4 .
$$

Thus, by Lemma 2.4, for $l=1,2,3$, we have

$$
\begin{aligned}
& I_{1_{1}}^{(l)}=\int_{E} \frac{1}{\left|\xi^{\prime}-\eta\right|^{(4-l) q}\left|\xi^{\prime \prime}-\eta\right|^{l q}} d E_{\eta} \\
& \leq M_{0}\left(\alpha_{l}, \beta_{l}\right)\left|\xi^{\prime}-\xi^{\prime \prime}\right|^{4-\alpha_{l}-\beta_{l}} \\
& =M_{0}\left(\alpha_{l}, \beta_{l}\right)\left|\xi^{\prime}-\xi^{\prime \prime}\right|^{4-4 q} \text {. }
\end{aligned}
$$

Thus, by inequalities (3.7) and (3.8), we obtain

$$
\begin{aligned}
I_{1_{1}} & \leq \frac{1}{2 \pi^{2}}\|g\|_{L_{p}}\left|\xi^{\prime}-\xi^{\prime \prime}\right| \sum_{l=1}^{3}\left(M_{0}\left(\alpha_{l}, \beta_{l}\right)\left|\xi^{\prime}-\xi^{\prime \prime}\right|^{4-4 q}\right)^{\frac{1}{q}} \\
& \leq J_{4}\|g\|_{L_{p}}\left|\xi^{\prime}-\xi^{\prime \prime}\right|^{\beta},
\end{aligned}
$$

where $0<\beta=1+(4-4 q) / q=1-4 / p<1$.

Next, we discuss $I_{1_{2}}$.

For arbitrary $\xi^{\prime}, \xi^{\prime \prime} \in \mathbb{C}^{2} \cong \mathbb{R}^{4}, \xi^{\prime} \neq \xi^{\prime \prime}$, we suppose $\left|\xi^{\prime}-\xi^{\prime \prime}\right|=\delta$ and construct a sphere $B\left(\xi^{\prime}, 3 \delta\right)$ with the center at $\xi^{\prime}$ and radius $3 \delta$. Next we discuss $I_{1_{2}}$ in two cases.

(i) If $B\left(\xi^{\prime}, 3 \delta\right) \cap \bar{E} \neq \emptyset$, then we may suppose $B\left(\xi^{\prime}, 3 \delta\right) \cap \bar{E}=\Omega_{1}, \bar{E}-\Omega_{1}=\Omega_{2}$. Thus we have

$$
\begin{aligned}
I_{1_{2}}= & \frac{1}{2 \pi^{2}} \int_{E} \mathcal{K}_{2}\left(\xi^{\prime}, \xi^{\prime \prime}, \eta\right)|g(\eta)| d E_{\eta} \\
= & \frac{1}{2 \pi^{2}} \int_{\Omega_{1}} \mathcal{K}_{2}\left(\xi^{\prime}, \xi^{\prime \prime}, \eta\right)|g(\eta)| d \Omega_{1 \eta} \\
& +\frac{1}{2 \pi^{2}} \int_{\Omega_{2}} \mathcal{K}_{2}\left(\xi^{\prime}, \xi^{\prime \prime}, \eta\right)|g(\eta)| d \Omega_{2 \eta} \\
= & I_{1_{2}}^{(1)}+I_{1_{2}}^{(2)} .
\end{aligned}
$$

Again, by inequality (3.4), the Hölder inequality, and the use of a local generalized spherical coordinate, we have

$$
\begin{aligned}
I_{1_{2}}^{(1)} \leq & \frac{1}{2 \pi^{2}} \int_{\Omega_{1}} \frac{\left|\bar{z}_{1}^{\prime}-\bar{\zeta}_{1}\right|\left|\xi^{\prime \prime}-\eta\right|^{2}\left|z_{2}^{\prime \prime}-\varsigma_{2}\right|^{2}}{\left|\xi^{\prime}-\eta\right|^{4}\left|\xi^{\prime \prime}-\eta\right|^{4}}|g(\eta)| d \Omega_{1 \eta} \\
& +\frac{1}{2 \pi^{2}} \int_{\Omega_{1}} \frac{\left|z_{2}^{\prime}-\varsigma_{2}\right|^{2}\left|\xi^{\prime}-\eta\right|^{2}\left|\bar{z}_{1}^{\prime \prime}-\bar{\zeta}_{1}\right|}{\left|\xi^{\prime}-\eta\right|^{4}\left|\xi^{\prime \prime}-\eta\right|^{4}}|g(\eta)| d \Omega_{1 \eta} \\
\leq & \frac{1}{2 \pi^{2}} \int_{\Omega_{1}} \frac{1}{\left|\xi^{\prime}-\eta\right|^{3}}|g(\eta)| d \Omega_{1 \eta}+\frac{1}{2 \pi^{2}} \int_{\Omega_{1}} \frac{1}{\left|\xi^{\prime \prime}-\eta\right|^{3}}|g(\eta)| d \Omega_{1 \eta} \\
\leq & J_{5}\|g\|_{L_{p}}\left[\left(\int_{\Omega_{1}} \frac{1}{\left|\xi^{\prime}-\eta\right|^{3 q}} d \Omega_{1 \eta}\right)^{\frac{1}{q}}+\left(\int_{\Omega_{1}} \frac{1}{\left|\xi^{\prime \prime}-\eta\right|^{3 q}} d \Omega_{1 \eta}\right)^{\frac{1}{q}}\right] \\
\leq & J_{6}\|g\|_{L_{p}}\left[\left(\int_{0}^{3 \delta} \frac{1}{\rho^{3 q-3}} d \rho\right)^{\frac{1}{q}}+\left(\int_{0}^{4 \delta} \frac{1}{\rho^{3 q-3}} d \rho\right)^{\frac{1}{q}}\right]
\end{aligned}
$$




$$
\begin{aligned}
& \leq J_{7}\|g\|_{L_{p}} \delta^{\frac{4-3 q}{q}}=J_{7}\|g\|_{L_{p}}\left|\xi^{\prime}-\xi^{\prime \prime}\right|^{1-\frac{4}{p}} \\
& =J_{7}\|g\|_{L_{p}}\left|\xi^{\prime}-\xi^{\prime \prime}\right|^{\beta} .
\end{aligned}
$$

In addition,

$$
\begin{aligned}
\mathcal{K}_{2}\left(\xi^{\prime}, \xi^{\prime \prime}, \eta\right) & \\
= & \frac{\left|\left(\bar{z}_{1}^{\prime}-\bar{\zeta}_{1}\right)\right| \xi^{\prime \prime}-\left.\eta\right|^{2}\left|z_{2}^{\prime \prime}-\varsigma_{2}\right|^{2}-\left|z_{2}^{\prime}-\varsigma_{2}\right|^{2}\left|\xi^{\prime}-\eta\right|^{2}\left(\bar{z}_{1}^{\prime \prime}-\bar{\zeta}_{1}\right) \mid}{\left|\xi^{\prime}-\eta\right|^{4}\left|\xi^{\prime \prime}-\eta\right|^{4}} \\
= & \frac{\left|\left(\bar{z}_{1}^{\prime \prime}-\bar{\zeta}_{1}+\bar{z}_{1}^{\prime}-\bar{z}_{1}^{\prime \prime}\right)\right| \xi^{\prime \prime}-\left.\eta\right|^{2}\left|z_{2}^{\prime \prime}-\varsigma_{2}\right|^{2}-\left|z_{2}^{\prime}-\varsigma_{2}\right|^{2}\left|\xi^{\prime}-\eta\right|^{2}\left(\bar{z}_{1}^{\prime \prime}-\bar{\zeta}_{1}\right) \mid}{\left|\xi^{\prime}-\eta\right|^{4}\left|\xi^{\prime \prime}-\eta\right|^{4}} \\
= & \mid \frac{\left.\mid \bar{z}_{1}^{\prime \prime}-\bar{\zeta}_{1}\right)\left|\xi^{\prime \prime}-\eta\right|^{2}\left|z_{2}^{\prime \prime}-\varsigma_{2}\right|^{2}+\left(\bar{z}_{1}^{\prime}-\bar{z}_{1}^{\prime \prime}\right)\left|\xi^{\prime \prime}-\eta\right|^{2}\left|z_{2}^{\prime \prime}-\varsigma_{2}\right|^{2}}{\left|\xi^{\prime}-\eta\right|^{4}\left|\xi^{\prime \prime}-\eta\right|^{4}} \\
& -\frac{\left(\bar{z}_{1}^{\prime \prime}-\bar{\zeta}_{1}\right)\left|z_{2}^{\prime}-\varsigma_{2}\right|^{2}\left|\xi^{\prime}-\eta\right|^{2} \mid}{\left|\xi^{\prime}-\eta\right|^{4}\left|\xi^{\prime \prime}-\eta\right|^{4}} \mid \\
\leq & \frac{\left|z_{1}^{\prime \prime}-\varsigma_{1}\right||| \xi^{\prime \prime}-\left.\eta\right|^{2}\left|z_{2}^{\prime \prime}-\varsigma_{2}\right|^{2}-\left|z_{2}^{\prime}-\varsigma_{2}\right|^{2}\left|\xi^{\prime}-\eta\right|^{2} \mid}{\left|\xi^{\prime}-\eta\right|^{4}\left|\xi^{\prime \prime}-\eta\right|^{4}} \\
& +\frac{\left|z_{1}^{\prime}-z_{1}^{\prime \prime}\right|\left|\xi^{\prime \prime}-\eta\right|^{2}\left|z_{2}^{\prime \prime}-\varsigma_{2}\right|^{2}}{\left|\xi^{\prime}-\eta\right|^{4}\left|\xi^{\prime \prime}-\eta\right|^{4}} .
\end{aligned}
$$

Again, because of

$$
\begin{aligned}
|| \xi^{\prime \prime} & -\left.\eta\right|^{2}\left|z_{2}^{\prime \prime}-\varsigma_{2}\right|^{2}-\left|z_{2}^{\prime}-\varsigma_{2}\right|^{2}\left|\xi^{\prime}-\eta\right|^{2} \mid \\
= & || \xi^{\prime \prime}-\left.\eta\right|^{2}\left|z_{2}^{\prime \prime}-\varsigma_{2}\right|^{2}-\left|\xi^{\prime \prime}-\eta\right|^{2}\left|z_{2}^{\prime}-\varsigma_{2}\right|^{2} \\
& \quad+\left|\xi^{\prime \prime}-\eta\right|^{2}\left|z_{2}^{\prime}-\varsigma_{2}\right|^{2}-\left|z_{2}^{\prime}-\varsigma_{2}\right|^{2}\left|\xi^{\prime}-\eta\right|^{2} \mid \\
\leq & \left|\xi^{\prime \prime}-\eta\right|^{2}|| z_{2}^{\prime \prime}-\left.\varsigma_{2}\right|^{2}-\left|z_{2}^{\prime}-\varsigma_{2}\right|^{2}|+| z_{2}^{\prime}-\left.\varsigma_{2}\right|^{2}|| \xi^{\prime \prime}-\left.\eta\right|^{2}-\left|\xi^{\prime}-\eta\right|^{2} \mid \\
\leq & \left|\xi^{\prime \prime}-\eta\right|^{2}\left|z_{2}^{\prime \prime}-z_{2}^{\prime}\right|\left(\left|z_{2}^{\prime \prime}-\varsigma_{2}\right|+\left|z_{2}^{\prime}-\varsigma_{2}\right|\right)+\left|z_{2}^{\prime}-\varsigma_{2}\right|^{2}\left|\xi^{\prime \prime}-\xi^{\prime}\right|\left(\left|\xi^{\prime \prime}-\eta\right|+\left|\xi^{\prime}-\eta\right|\right) \\
\leq & \left|\xi^{\prime \prime}-\xi^{\prime}\right| \sum_{m=0}^{3}\left|\xi^{\prime \prime}-\eta\right|^{3-m}\left|\xi^{\prime}-\eta\right|^{m} .
\end{aligned}
$$

Thus, we have

$$
\begin{aligned}
& \mathcal{K}_{2}\left(\xi^{\prime}, \xi^{\prime \prime}, \eta\right) \\
& \quad \leq \frac{\left|\xi^{\prime \prime}-\eta\right|\left|\xi^{\prime \prime}-\xi^{\prime}\right| \sum_{m=0}^{3}\left|\xi^{\prime \prime}-\eta\right|^{3-m}\left|\xi^{\prime}-\eta\right|^{m}+\left|\xi^{\prime}-\xi^{\prime \prime}\right|\left|\xi^{\prime \prime}-\eta\right|^{2}\left|\xi^{\prime \prime}-\eta\right|^{2}}{\left|\xi^{\prime}-\eta\right|^{4}\left|\xi^{\prime \prime}-\eta\right|^{4}} \\
& \quad=\left|\xi^{\prime}-\xi^{\prime \prime}\right|\left(\frac{2}{\left|\xi^{\prime}-\eta\right|^{4}}+\sum_{l=1}^{3} \frac{1}{\left|\xi^{\prime}-\eta\right|^{4-l}\left|\xi^{\prime \prime}-\eta\right|^{l}}\right) .
\end{aligned}
$$

So by (3.10) and (3.12), we obtain

$$
\begin{aligned}
I_{1_{2}}^{(2)} & =\frac{1}{2 \pi^{2}} \int_{\Omega_{2}} \mathcal{K}_{2}\left(\xi^{\prime}, \xi^{\prime \prime}, \eta\right)|g(\eta)| d \Omega_{2 \eta} \\
& \leq\left|\xi^{\prime}-\xi^{\prime \prime}\right| \frac{1}{\pi^{2}} \int_{\Omega_{2}} \frac{|g(\eta)|}{\left|\xi^{\prime}-\eta\right|^{4}} d \Omega_{2 \eta}
\end{aligned}
$$




$$
\begin{aligned}
& +\left|\xi^{\prime}-\xi^{\prime \prime}\right| \frac{1}{2 \pi^{2}} \int_{\Omega_{2}} \sum_{l=1}^{3} \frac{|g(\eta)|}{\left|\xi^{\prime}-\eta\right|^{4-l}\left|\xi^{\prime \prime}-\eta\right|^{l}} d \Omega_{2 \eta} \\
= & I_{1_{2}}^{(2)^{\prime}}+I_{1_{2}}^{(2)^{\prime \prime}} .
\end{aligned}
$$

First, similar to the method estimating $I_{1_{1}}$, we have

$$
I_{1_{2}}^{(2)^{\prime \prime}} \leq J_{8}\|g\|_{L_{p}}\left|\xi^{\prime}-\xi^{\prime \prime}\right|^{\beta}
$$

Second, when $\eta \in \Omega_{2},\left|\xi^{\prime}-\eta\right|>3 \delta,\left|\xi^{\prime \prime}-\eta\right|>2 \delta$. Thus we have

$$
\begin{aligned}
& 2 \delta \leq\left|\xi^{\prime}-\eta\right|-\left|\xi^{\prime}-\xi^{\prime \prime}\right| \leq\left|\xi^{\prime \prime}-\eta\right| \leq\left|\xi^{\prime}-\xi^{\prime \prime}\right|+\left|\xi^{\prime}-\eta\right|=\delta+\left|\xi^{\prime}-\eta\right| \\
& \delta \leq\left|\xi^{\prime \prime}-\eta\right|-\left|\xi^{\prime \prime}-\xi^{\prime}\right| \leq\left|\xi^{\prime}-\eta\right| \leq\left|\xi^{\prime}-\xi^{\prime \prime}\right|+\left|\xi^{\prime \prime}-\eta\right|=\delta+\left|\xi^{\prime \prime}-\eta\right| .
\end{aligned}
$$

So we know

$$
\frac{1}{2} \leq \frac{\left|\xi^{\prime}-\eta\right|}{\left|\xi^{\prime \prime}-\eta\right|} \leq \frac{3}{2}
$$

Thus, by (3.16), the Hölder inequality, and Lemma 2.4, we can obtain

$$
\begin{aligned}
I_{1_{2}}^{(2)^{\prime}} & =\left|\xi^{\prime}-\xi^{\prime \prime}\right| \frac{1}{\pi^{2}} \int_{\Omega_{2}} \frac{|g(\eta)|}{\left|\xi^{\prime}-\eta\right|^{4}} d \Omega_{2 \eta} \\
& \leq\left|\xi^{\prime}-\xi^{\prime \prime}\right| \frac{1}{\pi^{2}} \int_{\Omega_{2}} \frac{2|g(\eta)|}{\left|\xi^{\prime}-\eta\right|^{3}\left|\xi^{\prime \prime}-\eta\right|} d \Omega_{2 \eta} \\
& \leq J_{9}\|g\|_{L_{p}}\left|\xi^{\prime}-\xi^{\prime \prime}\right|^{1-\frac{4}{p}} \\
& =J_{9}\|g\|_{L_{p}}\left|\xi^{\prime}-\xi^{\prime \prime}\right|^{\beta}
\end{aligned}
$$

So, by (3.13), (3.14), and (3.17), we have

$$
I_{1_{2}}^{(2)} \leq J_{10}\|g\|_{L_{p}}\left|\xi^{\prime}-\xi^{\prime \prime}\right|^{\beta} .
$$

Therefore, by (3.10), (3.11), and (3.18), we can obtain

$$
I_{1_{2}} \leq J_{11}\|g\|_{L_{p}}\left|\xi^{\prime}-\xi^{\prime \prime}\right|^{\beta}
$$

(ii) If $B\left(\xi^{\prime}, 3 \delta\right) \cap \bar{E}=\emptyset$, then for arbitrary $\eta \in E$, we have $\left|\xi^{\prime}-\eta\right|>3 \delta$, $\left|\xi^{\prime \prime}-\eta\right|>2 \delta$. Thus similar to the method estimating $I_{1_{2}}^{(2)}$, we have

$$
I_{1_{2}} \leq J_{12}\|g\|_{L_{p}}\left|\xi^{\prime}-\xi^{\prime \prime}\right|^{\beta}
$$

So, by (3.19) and (3.20), we have

$$
I_{1_{2}} \leq J_{13}\|g\|_{L_{p}}\left|\xi^{\prime}-\xi^{\prime \prime}\right|^{\beta}, \quad \xi^{\prime}, \xi^{\prime \prime} \in \mathbb{R}^{4} \cong \mathbb{C}^{2},
$$

where $J_{13}=\max \left\{J_{11}, J_{12}\right\}$. 
Thus, to sum up, by (3.5), (3.9), and (3.21), we obtain

$$
I_{1} \leq J_{14}\|g\|_{L_{p}}\left|\xi^{\prime}-\xi^{\prime \prime}\right|^{\beta}, \quad \xi^{\prime}, \xi^{\prime \prime} \in \mathbb{R}^{4} \cong \mathbb{C}^{2}
$$

Similarly, we have

$$
I_{2} \leq J_{15}\|g\|_{L_{p}}\left|\xi^{\prime}-\xi^{\prime \prime}\right|^{\beta}, \quad \xi^{\prime}, \xi^{\prime \prime} \in \mathbb{R}^{4} \cong \mathbb{C}^{2}
$$

So, by (3.3), (3.22), and (3.23), we obtain

$$
O_{3} \leq M_{2}^{\prime}(p)\|g\|_{L_{p}}\left|\xi^{\prime}-\xi^{\prime \prime}\right|^{\beta}, \quad \xi^{\prime}, \xi^{\prime \prime} \in \mathbb{R}^{4} \cong \mathbb{C}^{2}
$$

where $M_{2}^{\prime}(p)=J_{14}+J_{15}$.

For $\eta \in \mathbb{C}^{2}-E$, we suppose that $\eta=\frac{\bar{\eta}^{\prime}}{\left|\eta^{\prime}\right|^{2}}$, then we have $\left|\eta^{\prime}\right| \leq 1$. Thus by $g \in L_{p}\left(\mathbb{C}^{2}, \mathbb{H}\right)$, similar to the proof as stated above, we have

$$
O_{4} \leq M_{2}^{\prime \prime}(p)\|g\|_{L_{p}}\left|\xi^{\prime}-\xi^{\prime \prime}\right|^{\beta}, \quad \xi^{\prime}, \xi^{\prime \prime} \in \mathbb{R}^{4} \cong \mathbb{C}^{2}
$$

Therefore, for arbitrary $\xi^{\prime}, \xi^{\prime \prime} \in \mathbb{C}^{2} \cong \mathbb{R}^{4}, \xi^{\prime} \neq \xi^{\prime \prime}$, we obtain

$$
\left|{ }^{\psi} T_{\mathbb{C}^{2}}[g]\left(\xi^{\prime}\right)-{ }^{\psi} T_{\mathbb{C}^{2}}[g]\left(\xi^{\prime \prime}\right)\right| \leq M_{2}(p)\|g\|_{L_{p}}\left|\xi^{\prime}-\xi^{\prime \prime}\right|^{\beta}, \quad \xi^{\prime}, \xi^{\prime \prime} \in \mathbb{R}^{4} \cong \mathbb{C}^{2}
$$

where $M_{2}(p)=M_{2}^{\prime}(p)+M_{2}^{\prime \prime}(p)$, i.e. ${ }^{\psi} T_{\mathbb{C}^{2}}[g] \in C_{\beta}\left(\mathbb{C}^{2}, \mathbb{H}\right) \cong C_{\beta}\left(\mathbb{R}^{4}, \mathbb{H}\right)(0<\beta<1)$.

(3) For arbitrary $\varphi \in C_{0}^{\infty}\left(\mathbb{C}^{2}, \mathbb{H}\right)$, there exists a bounded closed set $Q \subset \mathbb{C}^{2}$, such that $\overline{\operatorname{supp} \varphi} \subset \subset Q$. Thus, by $T_{\mathbb{C}^{2}}[g](\infty)=0$, Definition 2.1, Lemma 2.3, and the Fubini theorem, we have

$$
\begin{aligned}
& \int_{\mathbb{C}^{2}}[\varphi]^{\psi} D(\xi)^{\psi} T_{\mathbb{C}^{2}}[g](\xi) d_{\mathbb{C}^{2} \xi} \\
& =\lim _{d \rightarrow \infty} \int_{Q}[\varphi]^{\psi} D(\xi)^{\psi} T_{\mathbb{C}^{2}}[g](\xi) d Q_{\xi} \\
& =-\lim _{d \rightarrow \infty} \int_{Q}[\varphi]^{\psi} D(\xi) \int_{\mathbb{C}^{2}} \mathcal{K}_{\psi}(\eta-\xi) g(\eta) d_{\mathbb{C}^{2} \eta} d Q_{\xi} \\
& =\lim _{d \rightarrow \infty} \int_{\mathbb{C}^{2}} \int_{Q}[\varphi]^{\psi} D(\xi) \mathcal{K}_{\psi}(\xi-\eta) d Q_{\xi} g(\eta) d_{\mathbb{C}^{2} \eta} \\
& =\lim _{d \rightarrow \infty} \int_{\mathbb{C}^{2}}\left[\int_{\partial Q} \varphi(\xi) d \sigma_{\xi} \mathcal{K}_{\psi}(\xi-\eta)-\varphi(\eta)\right] g(\eta) d_{\mathbb{C}^{2} \eta} \\
& =-\int_{\mathbb{C}^{2}} \varphi(\eta) g(\eta) d_{\mathbb{C}^{2} \eta}=-\int_{\mathbb{C}^{2}} \varphi(\xi) g(\xi) d_{\mathbb{C}^{2} \xi},
\end{aligned}
$$

where $d=\sup _{\xi^{\prime}, \xi^{\prime \prime} \in Q}\left|\xi^{\prime}-\xi^{\prime \prime}\right|$. Hence, in the sense of generalized derivatives, we have ${ }^{\psi} D\left({ }^{\psi} T_{\mathbb{C}^{2}}[g]\right)(\xi)=g(\xi)$.

Remark 3.1 By the process of proof in Theorem 3.1, it is easy to show that ${ }^{\psi} T_{\mathbb{C}^{2}}^{(1)}[g]$, ${ }^{\psi} T_{\mathbb{C}^{2}}^{(2)}[g] \in C_{\beta}\left(\mathbb{C}^{2}, \mathbb{C}\right) \cong C_{\beta}\left(\mathbb{R}^{4}, \mathbb{C}\right)(0<\beta<1)$. 


\section{Integral representation of solution of the mixed boundary value problem for the inhomogeneous Cimmino system}

In this section, let $E=E_{1} \times E_{2}$ be a bounded domain, $\partial E_{m}(m=1,2)$ be simply closed curves in the $z_{m}$-plane, and $\partial E_{m} \in C_{\mu}^{(1)}, 0<\mu<1$. Without loss of generality, we may consider $\partial E_{m}=\left\{z_{m}|| z_{m} \mid=1\right\}$ and $E_{m}=\left\{z_{m}|| z_{m} \mid<1\right\}(m=1,2)$. Denote by $E_{m}^{+}, E_{m}^{-}$the inner domain and outer domain of $\partial E_{m}$, respectively, and $E^{++}=E_{1}^{+} \times E_{2}^{+}, E^{+-}=E_{1}^{+} \times E_{2}^{-}, E^{-+}=E_{1}^{-} \times E_{2}^{+}$, $E^{--}=E_{1}^{-} \times E_{2}^{-}, \Gamma=\partial E_{1} \times \partial E_{2}$.

Problem $\mathbf{P}$ The mixed boundary value problem for the inhomogeneous Cimmino system (1.2) is to find a function $f\left(z_{1}, z_{2}\right)=u_{1}\left(z_{1}, z_{2}\right)+u_{2}\left(z_{1}, z_{2}\right) j$ satisfying the Cimmino system (1.2) and the following boundary condition:

$$
\begin{aligned}
u_{1}^{++}\left(t_{1}, t_{2}\right)= & G_{1}\left(t_{1}, t_{2}\right) u_{1}^{+-}\left(t_{1}, t_{2}\right)+G_{2}\left(t_{1}, t_{2}\right) u_{1}^{-+}\left(t_{1}, t_{2}\right) \\
& +G_{3}\left(t_{1}, t_{2}\right) u_{1}^{--}\left(t_{1}, t_{2}\right)+H\left(t_{1}, t_{2}\right), \quad t=\left(t_{1}, t_{2}\right) \in \Gamma, \\
u_{2}\left(t_{1}, t_{2}\right)= & h\left(t_{1}, t_{2}\right), \quad t=\left(t_{1}, t_{2}\right) \in \partial E,
\end{aligned}
$$

where $u_{1}=f_{0}+i f_{1}, u_{2}=f_{2}+i f_{3}, z_{1}=x_{0}+i x_{1}, z_{2}=x_{2}+i x_{3} . G_{1}\left(z_{1}, z_{2}\right), G_{2}\left(z_{1}, z_{2}\right), G_{3}\left(z_{1}, z_{2}\right)$ are analytic in $E^{+-}, E^{-+}, E^{--}$and are continuous in $\bar{E}^{+-}, \bar{E}^{-+}, \bar{E}^{--}$, respectively, which have no zero. We have $G_{m}\left(t_{1}, t_{2}\right)(m=1,2,3), H\left(t_{1}, t_{2}\right) \in C_{\alpha}(\Gamma, \mathbb{C}), h\left(t_{1}, t_{2}\right) \in C_{\alpha}(\partial E, \mathbb{C})(0<\alpha<1)$.

Lemma 4.1 If $\Psi \in C^{(2)}(E, \mathbb{H}), h \in C_{\alpha}(\partial E, \mathbb{C})(0<\alpha<1), g \in L_{p}\left(\mathbb{C}^{2}, \mathbb{H}\right)(4<p<+\infty)$, then the equation ${ }^{\psi} D[\Psi]=0$ with the boundary condition $\left.\bar{w}_{2}\right|_{\partial E}=\bar{h}\left(t_{1}, t_{2}\right)-\overline{{ }^{\psi} T_{\mathbb{C}^{2}}^{(2)}[g]}\left(t_{1}, t_{2}\right)$ has the solution $\Psi=w_{1}+w_{2} j=w_{1}+j \bar{w}_{2}$ and

$$
\begin{aligned}
& \bar{w}_{2}(\xi)=\int_{\partial E}\left[\bar{h}(t)-\overline{\psi T_{\mathbb{C}^{2}}^{(2)}[g]}(t)\right] \frac{\partial}{\partial v} G(\xi, t) d \partial E_{t}, \\
& w_{1}(\xi)=\Phi(\xi)+w_{0}(\xi)
\end{aligned}
$$

or

$$
\begin{aligned}
& \bar{w}_{2}\left(z_{1}, z_{2}\right)=\int_{\partial E}\left[\bar{h}\left(t_{1}, t_{2}\right)-\overline{\psi T_{\mathbb{C}^{2}}^{(2)}[g]}\left(t_{1}, t_{2}\right)\right] \frac{\partial}{\partial v} G\left(z_{1}, z_{2}, t_{1}, t_{2}\right) d \partial E_{t_{1}, t_{2}}, \\
& w_{1}\left(z_{1}, z_{2}\right)=\Phi\left(z_{1}, z_{2}\right)+w_{0}\left(z_{1}, z_{2}\right),
\end{aligned}
$$

where $v$ is the unit outward normal on $\partial E, G(\xi, \eta)$ is the Green's function in $E=E_{1} \times E_{2}$, $\Phi\left(z_{1}, z_{2}\right)$ is an arbitrary analytic function in $E=E_{1} \times E_{2}$, and

$$
\begin{aligned}
& { }^{\psi} T_{\mathbb{C}^{2}}^{(2)}[g]\left(t_{1}, t_{2}\right)=\frac{1}{2 \pi^{2}} \int_{\mathbb{C}^{2}} \frac{\left(\bar{t}_{2}-\bar{\varsigma}_{2}\right)}{\left(\left|t_{1}-\varsigma_{1}\right|^{2}+\left|t_{2}-\varsigma_{2}\right|^{2}\right)^{2}} g\left(\varsigma_{1}, \varsigma_{2}\right) d_{\mathbb{C}_{\varsigma_{1}, \varsigma_{2}}^{2}}, \\
& w_{0}\left(z_{1}, z_{2}\right)=\widetilde{T}_{E_{1}}\left[-\partial_{z_{2}} \bar{w}_{2}\right]+\widetilde{T}_{E_{2}}\left[\Phi_{0}\right], \\
& \widetilde{T}_{E_{1}}\left[-\partial_{z_{2}} \bar{w}_{2}\right]=-\frac{1}{\pi} \int_{E_{1}} \frac{-\partial_{z_{2}} \bar{w}_{2}\left(\varsigma_{1}, z_{2}\right)}{\varsigma_{1}-z_{1}} d E_{1 \varsigma_{1}}, \\
& \widetilde{T}_{E_{2}}\left[\Phi_{0}\right]=-\frac{1}{\pi} \int_{E_{2}} \frac{\Phi_{0}\left(z_{1}, \varsigma_{2}\right)}{\varsigma_{2}-z_{2}} d E_{2 \varsigma_{2}}, \\
& \Phi_{0}\left(z_{1}, z_{2}\right)=\frac{1}{2 \pi i} \int_{\partial E_{1}} \frac{\partial_{z_{1}} \bar{w}_{2}\left(\varsigma_{1}, z_{2}\right)}{\varsigma_{1}-z_{1}} d \partial E_{1} \varsigma_{1} .
\end{aligned}
$$


Proof From Remark 3.1, we know ${ }^{\psi} T_{\mathbb{C}^{2}}^{(2)}[g] \in C_{\beta}\left(\mathbb{C}^{2}, \mathbb{C}\right) \cong C_{\beta}\left(\mathbb{R}^{4}, \mathbb{C}\right)(0<\beta<1)$. Thus by [9], we have $\bar{h}-\overline{\psi T_{\mathbb{C}^{2}}^{(2)}[g]} \in C_{\mu}(\partial E, \mathbb{C})(0<\mu=\min \{\alpha, \beta\}<1)$. So we may construct

$$
\bar{w}_{2}(\xi)=\int_{\partial E}\left[\bar{h}(t)-\overline{\psi T_{\mathbb{C}^{2}}^{(2)}[g]}(t)\right] \frac{\partial}{\partial \nu} G(\xi, t) d \partial E_{t},
$$

where $v$ is the unit outward normal on $\partial E, G(\xi, \eta)$ is the Green's function in $E=E_{1} \times E_{2}$, and

$$
{ }^{\psi} T_{\mathbb{C}^{2}}^{(2)}[g]\left(t_{1}, t_{2}\right)=\frac{1}{2 \pi^{2}} \int_{\mathbb{C}^{2}} \frac{\left(\bar{t}_{2}-\bar{\zeta}_{2}\right)}{\left(\left|t_{1}-\varsigma_{1}\right|^{2}+\left|t_{2}-\varsigma_{2}\right|^{2}\right)^{2}} g\left(\varsigma_{1}, \varsigma_{2}\right) d_{\mathbb{C}_{\varsigma_{1}, \varsigma_{2}}^{2}} .
$$

Then $\bar{w}_{2}(\xi)$ is a complex-value harmonic function in $E$, i.e. $\Delta_{\mathbb{C}^{2}} \bar{w}_{2}=4\left(\partial_{z_{1} \bar{z}_{1}}^{2}+\partial_{z_{2} \bar{z}_{2}}^{2}\right) \bar{w}_{2}=0$. Hence

$$
\partial_{\bar{z}_{1}}\left(\partial_{z_{1}} \bar{w}_{2}\right)=-\partial_{\bar{z}_{2}}\left(\partial_{z_{2}} \bar{w}_{2}\right) \text {. }
$$

Again, by (3.1), we have

$$
{ }^{\psi} D[\Psi]=0 \quad \Longleftrightarrow \quad \begin{aligned}
& \partial_{\bar{z}_{1}} w_{1}+\partial_{z_{2}} \bar{w}_{2}=0, \\
& \partial_{\bar{z}_{2}} w_{1}-\partial_{z_{1}} \bar{w}_{2}=0
\end{aligned} \Longleftrightarrow\left\{\begin{array}{l}
\partial_{\bar{z}_{1}} w_{1}=-\partial_{z_{2}} \bar{w}_{2} \\
\partial_{\bar{z}_{2}} w_{1}=\partial_{z_{1}} \bar{w}_{2} .
\end{array}\right.
$$

By (4.3), we know $-\partial_{z_{2}} \bar{w}_{2}, \partial_{z_{1}} \bar{w}_{2}$ satisfy the compatibility condition

$$
\partial_{\bar{z}_{2}}\left(-\partial_{z_{2}} \bar{w}_{2}\right)=\partial_{\bar{z}_{1}}\left(\partial_{z_{1}} \bar{w}_{2}\right)
$$

Thus by Theorem 7.2.1 of Chapter 7 in [11], the general solution $w_{1}\left(z_{1}, z_{2}\right)$ of system (4.4) possesses the form

$$
w_{1}\left(z_{1}, z_{2}\right)=\Phi\left(z_{1}, z_{2}\right)+w_{0}\left(z_{1}, z_{2}\right)
$$

where $\Phi\left(z_{1}, z_{2}\right)$ is an arbitrary analytic function in $E=E_{1} \times E_{2}$ and

$$
\begin{aligned}
& w_{0}\left(z_{1}, z_{2}\right)=\widetilde{T}_{E_{1}}\left[-\partial_{z_{2}} \bar{w}_{2}\right]+\widetilde{T}_{E_{2}}\left[\Phi_{0}\right], \\
& \widetilde{T}_{E_{1}}\left[-\partial_{z_{2}} \bar{w}_{2}\right]=-\frac{1}{\pi} \int_{E_{1}} \frac{-\partial_{z_{2}} \bar{w}_{2}\left(\varsigma_{1}, z_{2}\right)}{\varsigma_{1}-z_{1}} d E_{1 \varsigma_{1}}, \\
& \widetilde{T}_{E_{2}}\left[\Phi_{0}\right]=-\frac{1}{\pi} \int_{E_{2}} \frac{\Phi_{0}\left(z_{1}, \varsigma_{2}\right)}{\varsigma_{2}-z_{2}} d E_{2 \varsigma_{2}}, \\
& \Phi_{0}\left(z_{1}, z_{2}\right)=\frac{1}{2 \pi i} \int_{\partial E_{1}} \frac{\partial_{z_{1}} \bar{w}_{2}\left(\varsigma_{1}, z_{2}\right)}{\varsigma_{1}-z_{1}} d \partial E_{1} \varsigma_{1} .
\end{aligned}
$$

Lemma 4.2 Let $G_{m}(m=1,2,3), H, w_{0}, E$ etc. be as stated above. Find a sectionally analytic function $\Phi\left(z_{1}, z_{2}\right)$ in $E^{++}, E^{+-}, E^{-+}, E^{--}$, such that $\Phi\left(z_{1}, z_{2}\right)$ is continuous in $E^{++}, E^{+-}, E^{-+}$, $E^{--}$and satisfies the boundary condition

$$
\begin{aligned}
\Phi^{++}\left(t_{1}, t_{2}\right)= & G_{1}\left(t_{1}, t_{2}\right) \Phi^{+-}\left(t_{1}, t_{2}\right)+G_{2}\left(t_{1}, t_{2}\right) \Phi^{-+}\left(t_{1}, t_{2}\right) \\
& +G_{3}\left(t_{1}, t_{2}\right) \Phi^{--}\left(t_{1}, t_{2}\right)+\left(G_{1}+G_{2}+G_{3}-1\right)\left(w_{0}\left(t_{1}, t_{2}\right)\right. \\
& \left.+{ }^{\psi} T_{\mathbb{C}^{2}}^{(1)}[g]\left(t_{1}, t_{2}\right)\right)+H\left(t_{1}, t_{2}\right), \quad t=\left(t_{1}, t_{2}\right) \in \Gamma,
\end{aligned}
$$


where

$$
{ }^{\psi} T_{\mathbb{C}^{2}}^{(1)}[g]\left(t_{1}, t_{2}\right)=\frac{1}{2 \pi^{2}} \int_{\mathbb{C}^{2}} \frac{\left(\bar{t}_{1}-\bar{\zeta}_{1}\right)}{\left(\left|t_{1}-\varsigma_{1}\right|^{2}+\left|t_{2}-\varsigma_{2}\right|^{2}\right)^{2}} g\left(\varsigma_{1}, \varsigma_{2}\right) d_{\mathbb{C}_{\varsigma_{1}, \varsigma_{2}}^{2}} .
$$

Then the solution has the form

$$
\Phi\left(z_{1}, z_{2}\right)= \begin{cases}F\left(z_{1}, z_{2}\right), & z=\left(z_{1}, z_{2}\right) \in E^{++} \\ F\left(z_{1}, z_{2}\right) / G_{1}\left(z_{1}, z_{2}\right), & z=\left(z_{1}, z_{2}\right) \in E^{+-} \\ F\left(z_{1}, z_{2}\right) / G_{2}\left(z_{1}, z_{2}\right), & z=\left(z_{1}, z_{2}\right) \in E^{-+} \\ -F\left(z_{1}, z_{2}\right) / G_{3}\left(z_{1}, z_{2}\right), & z=\left(z_{1}, z_{2}\right) \in E^{--}\end{cases}
$$

where

$$
F\left(z_{1}, z_{2}\right)=\frac{1}{(2 \pi i)^{2}} \int_{\partial E_{1} \times \partial E_{2}} \frac{\widetilde{H}\left(\varsigma_{1}, \varsigma_{2}\right)}{\left(\varsigma_{1}-z_{1}\right)\left(\varsigma_{2}-z_{2}\right)} d \partial E_{1 \varsigma_{1}} d \partial E_{2 \varsigma_{2}}
$$

and $\widetilde{H}=\left(G_{1}+G_{2}+G_{3}-1\right)\left(w_{0}+{ }^{\psi} T_{\mathbb{C}^{2}}^{(1)}[g]\right)+H$.

Proof From Remark 3.1, we know ${ }^{\psi} T_{\mathbb{C}^{2}}^{(1)}[g] \in C_{\beta}\left(\mathbb{C}^{2}, \mathbb{C}\right) \cong C_{\beta}\left(\mathbb{R}^{4}, \mathbb{C}\right)(0<\beta<1)$. Thus by [9], we have $\widetilde{H}=\left(G_{1}+G_{2}+G_{3}-1\right)\left(w_{0}+{ }^{\psi} T_{\mathbb{C}^{2}}^{(1)}[g]\right)+H \in C_{\mu}(\Gamma, \mathbb{C})(0<\mu=\min \{\alpha, \beta\}<1)$. Hence by Theorem 7.1.2 of Chapter 7 in [11], it is not difficult to verify this lemma.

Theorem 4.1 Let $E$, $\partial E$ etc. be as stated above. If $g \in L_{p}\left(\mathbb{C}^{2}, \mathbb{H}\right)(4<p<+\infty)$, then the solution of Problem $\mathrm{P}$ can be expressed as

$$
f(\xi)=\Psi(\xi)+{ }^{\psi} T_{\mathbb{C}^{2}}[g](\xi),
$$

where ${ }^{\psi} D[\Psi]=0$ and

$$
\begin{aligned}
& \left\{\begin{array}{l}
\Psi(\xi)=w_{1}(\xi)+w_{2}(\xi) j=w_{1}(\xi)+j \bar{w}_{2}(\xi), \\
w_{1}(\xi)=\Phi(\xi)+w_{0}(\xi), \\
\bar{w}_{2}(\xi)=\int_{\partial E}\left[\bar{h}(t)-\bar{\psi}_{T_{\mathbb{C}^{2}}^{(2)}[g]}(t)\right] \frac{\partial}{\partial \nu} G(\xi, t) d \partial E_{t},
\end{array}\right. \\
& { }^{\psi} T_{\mathbb{C}^{2}}[g](\xi)={ }^{\psi} T_{\mathbb{C}^{2}}[g]\left(z_{1}, z_{2}\right)=\frac{1}{2 \pi^{2}} \int_{\mathbb{C}^{2}} \frac{\left(\bar{z}_{1}-\bar{\zeta}_{1}\right)+\left(\bar{z}_{2}-\bar{\zeta}_{2}\right) j}{\left(\left|z_{1}-\varsigma_{1}\right|^{2}+\left|z_{2}-\varsigma_{2}\right|^{2}\right)^{2}} g\left(\varsigma_{1}, \varsigma_{2}\right) d_{\mathbb{C}_{1}^{2}, \varsigma_{2}} \\
& ={ }^{\psi} T_{\mathbb{C}^{2}}^{(1)}[g]\left(z_{1}, z_{2}\right)+{ }^{\psi} T_{\mathbb{C}^{2}}^{(2)}[g]\left(z_{1}, z_{2}\right) j,
\end{aligned}
$$

herein $w_{0},{ }^{\psi} T_{\mathbb{C}^{2}}^{(2)}[g]$ are as stated in Lemma $4.1, \Phi,{ }^{\psi} T_{\mathbb{C}^{2}}^{(1)}[g]$ are as stated in Lemma 4.2.

Proof By Theorem 3.1, we know ${ }^{\psi} D\left[{ }^{\psi} T_{\mathbb{C}^{2}}[g]\right](\xi)=g(\xi)$, thus ${ }^{\psi} D\left[\Psi(\xi)+{ }^{\psi} T_{\mathbb{C}^{2}}[g](\xi)\right]=g(\xi)$. Hence, by (3.2), we know the general solution of system (1.2) has the form

$$
f(\xi)=\Psi(\xi)+{ }^{\psi} T_{\mathbb{C}^{2}}[g](\xi),
$$

where ${ }^{\psi} D[\Psi]=0, \xi=z_{1}+z_{2} j, f(\xi)=f\left(z_{1}, z_{2}\right)=u_{1}\left(z_{1}, z_{2}\right)+u_{2}\left(z_{1}, z_{2}\right) j=u_{1}\left(z_{1}, z_{2}\right)+j \bar{u}_{2}\left(z_{1}, z_{2}\right)$, $\Psi(\xi)=\Psi\left(z_{1}, z_{2}\right)=w_{1}\left(z_{1}, z_{2}\right)+w_{2}\left(z_{1}, z_{2}\right) j=w_{1}\left(z_{1}, z_{2}\right)+j \bar{w}_{2}\left(z_{1}, z_{2}\right)$, and

$$
\begin{aligned}
& { }^{\psi} T_{\mathbb{C}^{2}}[g](\xi)={ }^{\psi} T_{\mathbb{C}^{2}}[g]\left(z_{1}, z_{2}\right)=\frac{1}{2 \pi^{2}} \int_{\mathbb{C}^{2}} \frac{\left(\bar{z}_{1}-\bar{\varsigma}_{1}\right)+\left(\bar{z}_{2}-\bar{\varsigma}_{2}\right) j}{\left(\left|z_{1}-\varsigma_{1}\right|^{2}+\left|z_{2}-\varsigma_{2}\right|^{2}\right)^{2}} g\left(\varsigma_{1}, \varsigma_{2}\right) d_{\mathbb{C}_{\varsigma_{1}, \varsigma_{2}}^{2}} \\
& ={ }^{\psi} T_{\mathbb{C}^{2}}^{(1)}[g]\left(z_{1}, z_{2}\right)+{ }^{\psi} T_{\mathbb{C}^{2}}^{(2)}[g]\left(z_{1}, z_{2}\right) j={ }^{\psi} T_{\mathbb{C}^{2}}^{(1)}[g]\left(z_{1}, z_{2}\right)+\overline{j^{\psi} T_{\mathbb{C}^{2}}^{(2)}[g]}\left(z_{1}, z_{2}\right) .
\end{aligned}
$$


Thus

$$
\bar{u}_{2}\left(z_{1}, z_{2}\right)=\bar{w}_{2}\left(z_{1}, z_{2}\right)+\overline{\psi T_{\mathbb{C}^{2}}^{(2)}[g]}\left(z_{1}, z_{2}\right)
$$

So the boundary condition (4.2) in Problem P can be written as

$$
\bar{w}_{2}=\bar{h}\left(t_{1}, t_{2}\right)-\overline{\psi T_{\mathbb{C}^{2}}^{(2)}[g]}\left(t_{1}, t_{2}\right), \quad t=\left(t_{1}, t_{2}\right) \in \partial E .
$$

Therefore, by Lemma 4.1, the solution to the equation ${ }^{\psi} D[\Psi]=0$ with boundary condition (4.8) can be expressed as

$$
\Psi(\xi)=w_{1}(\xi)+w_{2}(\xi) j=w_{1}(\xi)+j \bar{w}_{2}(\xi)
$$

where $w_{1}, \bar{w}_{2}$ are as stated in Lemma 4.1. Again, by (4.7), we have

$$
u_{1}\left(z_{1}, z_{2}\right)=w_{1}\left(z_{1}, z_{2}\right)+{ }^{\psi} T_{\mathbb{C}^{2}}^{(1)}[g]\left(z_{1}, z_{2}\right)
$$

From Lemma 4.1, we have

$$
w_{1}\left(z_{1}, z_{2}\right)=\Phi\left(z_{1}, z_{2}\right)+w_{0}\left(z_{1}, z_{2}\right)
$$

where $\Phi\left(z_{1}, z_{2}\right)$ is an arbitrary analytic function in $E=E_{1} \times E_{2}, w_{0}$ is as stated in Lemma 4.1. In addition, by Chapter 7 in [11], we know $w_{0} \in C_{\alpha}\left(\mathbb{C}^{2}, \mathbb{C}\right)(0<\alpha<1)$, by Remark 3.1, we know ${ }^{\psi} T_{\mathbb{C}^{2}}^{(1)}[g] \in C_{\beta}\left(\mathbb{C}^{2}, \mathbb{C}\right)(0<\beta<1)$. So the boundary condition (4.1) in Problem $\mathrm{P}$ can be written as

$$
\begin{aligned}
\Phi^{++}\left(t_{1}, t_{2}\right)= & G_{1}\left(t_{1}, t_{2}\right) \Phi^{+-}\left(t_{1}, t_{2}\right)+G_{2}\left(t_{1}, t_{2}\right) \Phi^{-+}\left(t_{1}, t_{2}\right) \\
& +G_{3}\left(t_{1}, t_{2}\right) \Phi^{--}\left(t_{1}, t_{2}\right)+\left(G_{1}+G_{2}+G_{3}-1\right)\left(w_{0}\left(t_{1}, t_{2}\right)\right. \\
& \left.+{ }^{\psi} T_{\mathbb{C}^{2}}^{(1)}[g]\left(t_{1}, t_{2}\right)\right)+H\left(t_{1}, t_{2}\right), \quad t=\left(t_{1}, t_{2}\right) \in \Gamma .
\end{aligned}
$$

Therefore, by Lemma 4.2, we know $\Phi\left(z_{1}, z_{2}\right)$ can be expressed as (4.6) in Lemma 4.2. In conclusion, we complete the proof.

The authors declare that they have no competing interests.

Authors' contributions

LPW has presented the main purpose of the article. All authors read and approved the final manuscript.

\section{Author details}

'College of Mathematics and Information Science, Hebei Normal University, Shijiazhuang, Hebei province, 050024,

P.R. China. ${ }^{2}$ School of Information, Renmin University of China, Beijing, 100872, P.R. China.

\section{Acknowledgements}

This work was supported by the National Science Foundation of China (No. 11401162, No. 11171349, No. 11301136), the Natural Science Foundation of Hebei Province (No. A2015205012, No. A2014205069, No. A2014208158) and Hebei Normal University Dr. Fund (No. L2015B03, No. L2015B04).

Received: 3 September 2014 Accepted: 22 December 2014 Published online: 30 January 2015 


\section{References}

1. Gürlebeck, K, Sprössig, W: Quaternionic and Clifford Calculus for Physicists and Engineers. Wiley, New York (1997)

2. Adler, SL: Quaternionic quantum field theory. Commun. Math. Phys. 104, 611-656 (1986)

3. Sprössig, W: Quaternionic operator methods in fluid dynamics. Adv. Appl. Clifford Algebras 18, $963-978$ (2008)

4. Alesker, S: Quaternionic Monge-Ampère equations. J. Geom. Anal. 13, 205-238 (2003)

5. Yang, PW, Li, D: $H_{\lambda}$-Regular vector functions and their boundary value problems. Bound. Value Probl. 2012,75 (2012)

6. Cimmino, G: Su alcuni sistemi lineari omogenei di equazioni alle derivate parziali del primo ordine. Rend. Semin. Mat. Univ. Padova 12, 89-113 (1941)

7. Dragomir, S, Lanconelli, E: On first order linear PDE systems all of whose solutions are harmonic functions. Tsukuba J. Math. 30, 149-170 (2006)

8. Abreu Blaya, R, Bory Reyes, J, Guzmán Adán, A, Schneider, B: Boundary value problems for the Cimmino system via quaternionic analysis. Appl. Math. Comput. 219, 3872-3881 (2012)

9. Iftimie, V: Functions hypercomplexes. Bull. Math. Soc. Sci. Math. Roum. 57, 279-332 (1965)

10. Gilbert, RP, Buchanan, JL: First Order Elliptic Systems: A Function Theoretic Approach. Academic Press, New York (1983)

11. Wen, GC: Recent Progress in Theory and Applications of Modern Complex Analysis. Science Press, Beijing (2010)

\section{Submit your manuscript to a SpringerOpen ${ }^{\ominus}$ journal and benefit from:}

- Convenient online submission

- Rigorous peer review

Immediate publication on acceptance

- Open access: articles freely available online

- High visibility within the field

- Retaining the copyright to your article 